\title{
Application of Large Eddy Simulation in the process of the Multi-Physics Field Coupling in a Combustion Chamber
}

\author{
Rang-Shu $X U^{1, a}$, Kai WANG ${ }^{1}$, Long $X U^{2}$ and $X i n ~ Y U^{2}$ \\ ${ }^{1}$ Shenyang Aerospace University, Aero-Engine Numerical Simulation Laboratory, Shenyang, China \\ ${ }^{2}$ Jinan Diesel Engine Co.,Ltd , No. 11966 JingShi Street, ChangQing District, Jinan, China
}

\begin{abstract}
Numerical simulation is the main method to solve turbulence problems. As one of the three methods which are commonly used in large-eddy simulation model (LES) is the most effective and promising research method. The basic idea of large-eddy simulation is that the large scale turbulent motion is directly simulated and we use the subgrid scale model to simulate small-scale turbulent motion. Continuing alternative load exists in aero-engine combustion chamber during operation. This coupling phenomenon is an important reason to the combustion chamber fatigue failure. In this paper, the large-eddy simulation methods are described and applied in researching aero-engine combustion chamber multi-physics field coupling analysis. By comparing with the experimental results we verify the feasibility of this method and there is great significance of actual project.

Keywords: Large Eddy Simulation; turbulence; Combustion Chamber; Multi-Physics Field Coupling; FEM.
\end{abstract}

\section{INTRODUCTION}

Turbulence is a kind of common natural phenomenon whose complexities are consist of randomness, rotation and statistics of motion, but it is difficult to solve turbulence problems because of its nonlinearity and pulsatory extreme wide frequency spectrum domain $[1,2]$. Numerical simulation and experiments are the main methods used in researching turbulence at present. Following the high speed development of computer technology large-eddy simulation has become the optimal method to solve turbulence problems in that it is able to save a mass of time and computer memory in contrast with direct numerical simulation and deal with insufficient information in RANS method [3], so a growing number of researchers favor it. The large-eddy simulation and the famous Smagorinsky model were suggested by Smagorinsky J.S. in 1963. In 1970s largeeddy simulation was used to solve hydraulic problems, it was improved by most researchers in 1980s and they put their attention to large-eddy simulation once more from the beginning of 1990s. From combustion to the flow around a circular cylinder till the phenomenon of vortex motion, large-eddy simulation is of irreplaceable functions and commonly used in projects [4].

Combustion chambers are one of the most important parts of aero-engines. There are considerable turbulent motions in the combustion process which is unstable because the ever-changing burning velocity results in pulsation phenomenon that can generate the speed origin and have an effect on the temperature field and generate the sonic pressure field at the same time. The small change of heat release speed can induce sound pressure perturbation, the spreading sound in combustor returns when to encounter the wall or different media and affect the fuel supply speed later. Heat release and sound pressure fluctuation interactive when their phase positions both get proper value and form a feedback circuit, thus, the system tends to be unstable. The tiny perturbation is enlarged in a short time, the shock with special amplitude and frequency is generated in a nonlinear condition afterwards. So, it becomes a coupling effect among heat of combustion, sound pressure and structure[5]. This multi-physics field coupling works on thin-wall construction of the engine combustors whose functions are greatly influenced and reduce the combustion efficiency, fatigue rupture occurs even have a strong impact on lifespan of the whole engine.

In this article, we make full use of large-eddy simulation method in researching Multi-Physics Coupling by finite element analysis that has important value in practical engineering application.

\section{Correlation Theory}

Multi-physics field coupling governing equations include mass equations, momentum equations and energy equations of the fluid and solid heat conduction equations as well as structural vibration equations.

The fluid mass equation is

$$
\frac{\partial \rho}{\partial t}+\frac{\partial\left(\rho u_{x}\right)}{\partial x}+\frac{\partial\left(\rho u_{y}\right)}{\partial y}+\frac{\partial\left(\rho u_{z}\right)}{\partial z}=0
$$

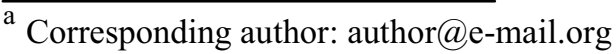


The fluid momentum equations are

$$
\left\{\begin{array}{l}
\frac{\partial\left(\rho u_{x}\right)}{\partial t}+\nabla \cdot\left(\rho u_{x} \vec{u}\right)=-\frac{\partial p}{\partial x}+\frac{\partial \tau_{x x}}{\partial x}+\frac{\partial \tau_{y x}}{\partial y}+\frac{\partial \tau_{z x}}{\partial z}+\rho f_{x} \\
\frac{\partial\left(\rho u_{y}\right)}{\partial t}+\nabla \cdot\left(\rho u_{y} \vec{u}\right)=-\frac{\partial p}{\partial y}+\frac{\partial \tau_{x y}}{\partial x}+\frac{\partial \tau y}{\partial y}+\frac{\partial \tau_{z y}}{\partial z}+\rho f_{y} \\
\frac{\partial\left(\rho u_{z}\right)}{\partial t}+\nabla \cdot\left(\rho u_{z} \vec{u}\right)=-\frac{\partial p}{\partial z}+\frac{\partial \tau_{x z}}{\partial x}+\frac{\partial \tau_{y z}}{\partial y}+\frac{\partial \tau_{z z}}{\partial z}+\rho f_{z}
\end{array}\right.
$$

The fluid energy equation is

$$
\frac{\partial(\rho E)}{\partial t}+\nabla \cdot[\vec{u}(\rho E+p)]=\nabla \cdot\left[k_{e f f} \nabla T-\sum_{j} h_{j} J_{j}+\left(\tau_{e f f} \cdot \vec{u}\right)\right]+S_{h}
$$

The massive structure of the combustor model belong to thin wall construction, by finite element analysis we can get the structural vibration equation

$$
M_{\varepsilon} \ddot{U}+C_{\varepsilon} \dot{U}+K_{\varepsilon} U=F_{p}+F_{o}
$$

In this equation, $r$ is displacement vector, $C_{s}$ is structural damping matrix, $K_{s}$ is structural stiffness matrix, $f_{p}$ is hydrokinetic knot vector on the interface between the fluid and solid, $f_{0}$ stands for the incentive vector.

After we take the acoustic discrete equations into account that we can get the finite element equation of the complete fluid-structure interaction:

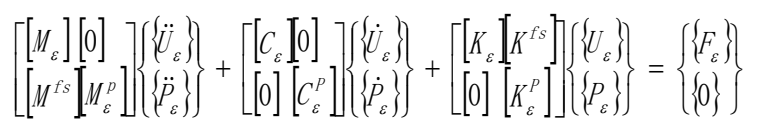

\section{Flow Fields Simulation}

Having applied the finite element method we built and meshed the combustor model by Ansys Workbench14.0, the model overall dimension depends on actual size of some type of aero-engine combustion liner whose total length is $491 \mathrm{~mm}$. The model we simplified likes fig.1.

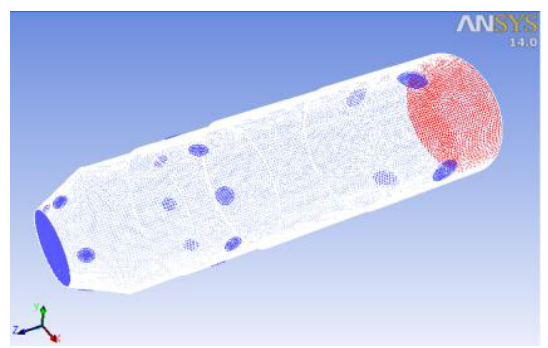

Fig.1 The model grid of combustion chamber

Then, we computed inner flow field of the combustion chamber by using FLUENT, input the mesh, set relevant parameters and adopt species transport model and we took the large-eddy simulation in turbulence. The results are shown in fig. 2 .

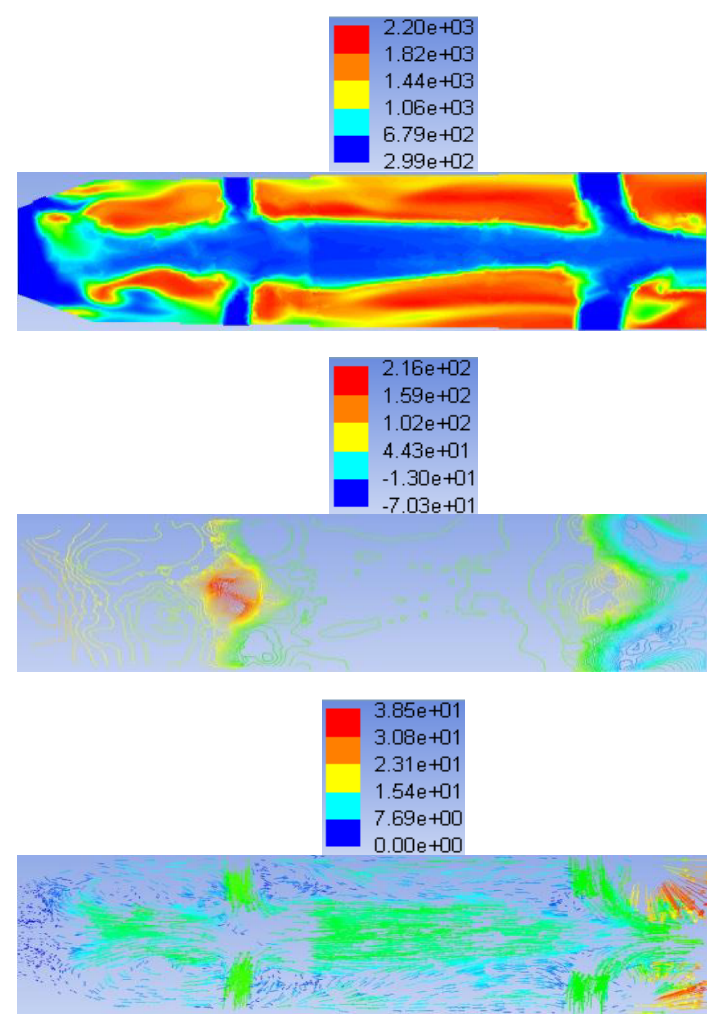

Fig.2 The flow distribution of temperature, pressure and velocity in the $\mathrm{Y}=0 \mathrm{~mm}$ plane

From fig. 2 and fig. 3 we can see that the inner pressure field and the speed field are influenced by temperature distribution and there is a high reflow area in the combustion chamber. These regional flow fields change and impact on noise propagation and the formation of multi-physics field coupling.
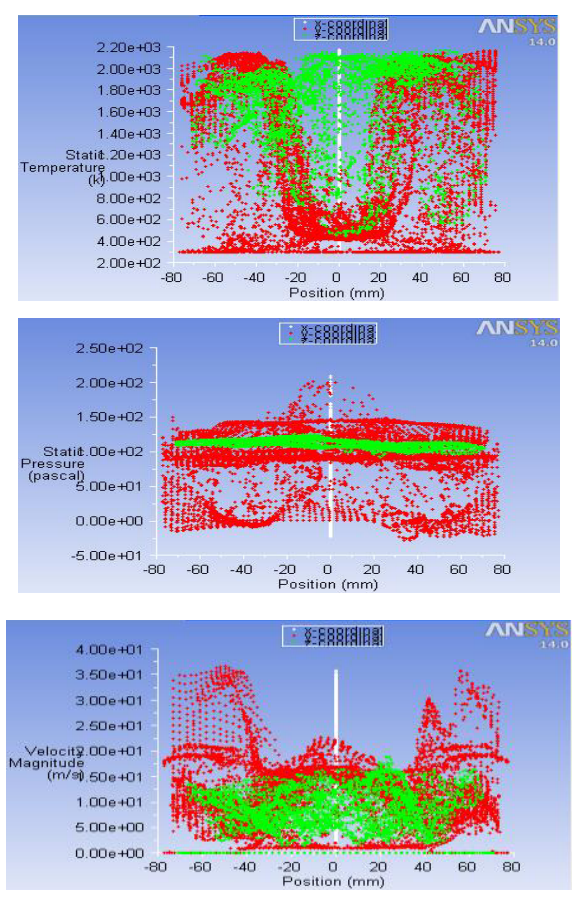

Fig.3 The central lines of temperature, pressure and velocity 


\section{Computation of Structural Dynamics.}

To start combustion calculation after computed the cold state. We got states similar to actual inner temperature field, sound pressure field and speed field by simulating combustion and import the results to the model of combustor, here are static results in fig. 4 and fig.5.

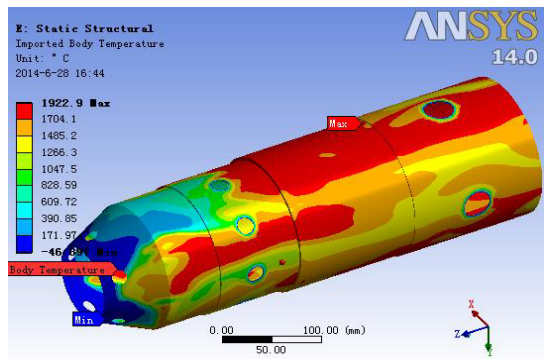

Fig.4 Import the temperature loads

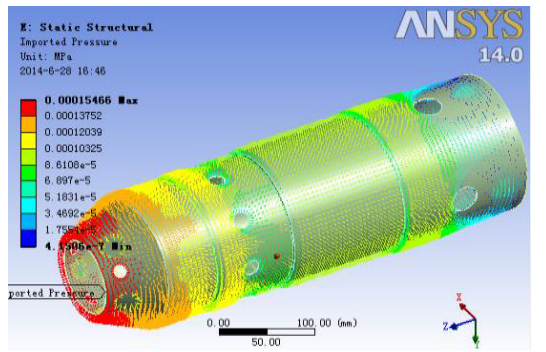

Fig.5 Import the pressure loads

Fig.6 depicts that the structural deformation pattern and center line temperature profile of the combustion chamber got by using large-eddy simulation method. Flame tube is fixed-fixed.
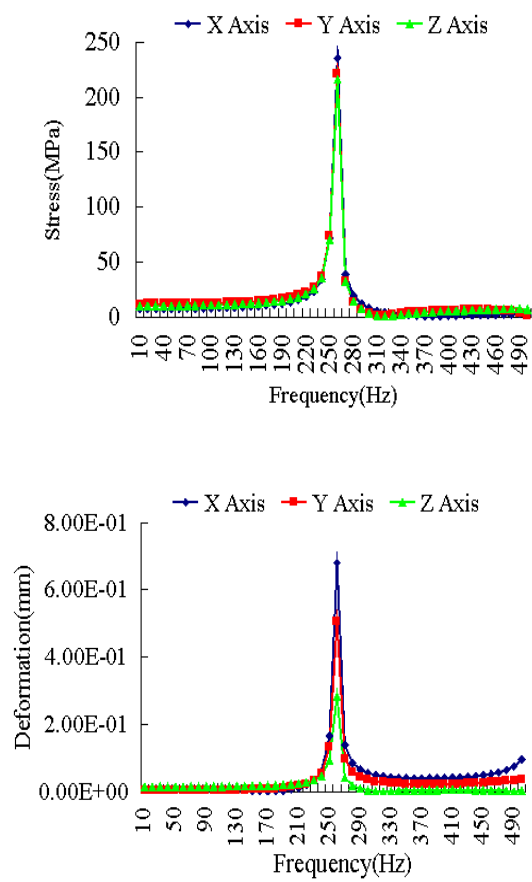

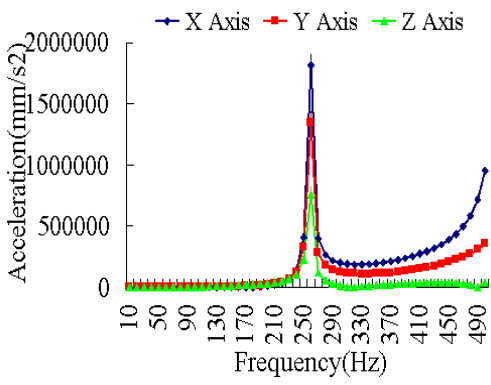

Fig.6 The harmonic response analysis

\section{Summary}

In the article we introduced the large-eddy simulation method and the multi-physics field coupling at first and then we simulated the model built by using Ansys Workbench 14.0 and analyzed this coupling later, by analyzing and computing this coupling we get the conclusion like that:

1)Comparing with the other two numerical simulation methods large-eddy simulation method has obvious advantages, it is able to analyze the coupling.

2)The computed results suggest that the coupling increase amplitude and reduce structural vibration frequency.

3)By comparing with experimental results we can see that using large-eddy simulation method can get better results.

\section{References}

1. F. Beaubert, S. Viazzo. Large eddy simulations of plane turbulent impinging jets at moderate Reynolds numbers $[\mathrm{J}]$. International Journal of Heat and Fluid Flow, 2003.

2. Roland Bouffanais. Advances and challenges of applied large-eddy simulation[J]. Computers \& Fluids, 2010.

3. Rob. Huls. Acousto-Elastic Interaction in Combustion Chambers[D]. Netherlands. University of Twente, Enschede. May 2006.

4. R.A.Huls. Vibration prediction in combustion chambers by coupling finite elements and large eddy simulations. Journal of Sound and Vibration 304 (2007) 224-229

5. D.J.Bodony, S.K.Lele. Low frequency sound sources in high-speed turbulent jets[R]. Center for Turbulence Research's Annual Research Briefs. 2006. 